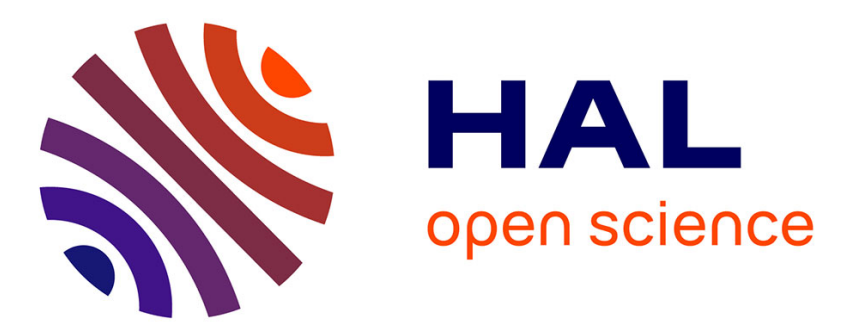

\title{
Research on Detection Moisture of Intact Meat Based on Discrete LED Wavelengths
}

\author{
Li-Feng Fan, Jian-Xu Wang, Peng-Fei Zhao, Hao Li, Zhong-Yi Wang, Lan \\ Huang
}

\section{- To cite this version:}

Li-Feng Fan, Jian-Xu Wang, Peng-Fei Zhao, Hao Li, Zhong-Yi Wang, et al.. Research on Detection Moisture of Intact Meat Based on Discrete LED Wavelengths. 8th International Conference on Computer and Computing Technologies in Agriculture (CCTA), Sep 2014, Beijing, China. pp.411-418, 10.1007/978-3-319-19620-6_46 . hal-01420256

\section{HAL Id: hal-01420256 \\ https://hal.inria.fr/hal-01420256}

Submitted on 20 Dec 2016

HAL is a multi-disciplinary open access archive for the deposit and dissemination of scientific research documents, whether they are published or not. The documents may come from teaching and research institutions in France or abroad, or from public or private research centers.
L'archive ouverte pluridisciplinaire HAL, est destinée au dépôt et à la diffusion de documents scientifiques de niveau recherche, publiés ou non, émanant des établissements d'enseignement et de recherche français ou étrangers, des laboratoires publics ou privés.

\section{(c)(1)}

Distributed under a Creative Commons Attribution| 4.0 International License 


\title{
Research on detection moisture of intact meat based on discrete LED wavelengths
}

\author{
Li-Feng Fan ${ }^{1,3}$, Jian-Xu Wang ${ }^{1,2}$, Peng-Fei Zhao ${ }^{1,2}$,Hao Li $^{1}$,Zhong-Yi Wang ${ }^{1,2,3}$,Lan \\ Huang ${ }^{1,2,3, *}$
}

${ }^{1}$ College of Information and Electrical Engineering, China Agricultural University, Beijing 100083,China; ${ }^{2}$ Modern Precision Agriculture System Integration Research Key Laboratory of Ministry of Education, Beijing 100083, China ; ${ }^{3}$ Key Laboratory of Agricultural information acquisition technology (Beijing), Ministry of Agriculture, Beijing 100083, China;

*biomed hl@263.net, hlan@cau.edu.cn

\begin{abstract}
The existing researches focused on using the commercial full wavelength spectrometer to determine the quality parameters of meat by detecting the meat emulsion, which were difficult to achieve online and nondestructive detection of the moisture content of intact meat. Moreover, the accuracy of pieces moisture detection is low, and people did not consider differences in the organizational structure of the pork meat itself. In this paper, we have developed a portable data acquisition system based on discrete wavelengths of spectral, and used it to detect the moisture content of fresh intact pork meat within a certain depth range. Based on the steady-state spatially resolved spectroscopy and considering the muscle fiber structure and direction of intact pork meat, we have designed a device with a symmetrical structure, which has a wavelength of $1300 \mathrm{~nm}, 1450 \mathrm{~nm}, 1550 \mathrm{~nm}$ and $970 \mathrm{~nm}$ LED light source for detecting the moisture content of the samples obtained from the Longissimus, within a certain depth range, and verified the stability and linearity of the system. The results show that the coefficient of determination is 0.49 , and the detection range is $73.19 \% \sim 77.654 \%$. This study shows that scattering properties of meat is one of the main factors affecting the stability of detection.
\end{abstract}

Keywords: near infrared spectroscopy, waste cooking oil, support vector machine, parameters optimization

\section{Introduction}

China is a great country of pork production and consumption. With the improvement of living standards, the requirement of the quality of fresh meat is increasing, and the moisture content of fresh meat is an important quality trait, which impact on fresh meat processing, storage, transportation ${ }^{[1]}$.A high moisture content of fresh meat will accelerate the reproduction of bacteria and mold, which cause spoilage. Moisture content of fresh meat is not only an important attribute for consumers, but also affect the eating quality and nutritional quality of the meat ${ }^{[2]}$. Traditional detection methods such as sensory judgment and chemical testing, which are time consuming, more destructive, low efficiency, then it is necessary to develop the rapid detection technology and equipment research of pork quality, and realize the objective evaluation of pork quality. 
As a fast, economic, non-destructive, real-time detection method, near infrared spectroscopy (NIR) has been used widely in the field of food in recent years. At present, many experts and scholars applied near-infrared spectral analysis to measure pork moisture detection research ${ }^{[3-6]}$. There have been many reports about water detection of mincemeat which have good results. Isaksson ${ }^{[3]}$ et al has used an online detection system of mincemeat based on near-infrared reflectometer to detect the content of fat, protein and moisture in the minced beef. For pork meat moisture detection, with fresh pork as samples, $\mathrm{Ji}^{[7-9]}$ et al used near infrared discrete illuminant based on the steady state spatial resolution method of diffuse reflection which can solve the problem of detecting depth, to test moisture content of fresh meat, and got better experimental results.

Thus, the aim of this paper is to investigate the arrangement of light source and detector in a probe, and develop a device for detecting intact pork meat and establish a model to predict the moisture of meat.

\section{Principle and measuring device}

\subsection{Measuring principle}

The steady-state spatial resolution technology is based on the diffusion approximation theory, when the steady light source continuously penetrates a semi-infinite medium, the transmission of light within the medium is independent on the time, whose propagation path appears as a banana shape. See figure 1.The detecting depth is related to the space between light source and the detector. The greater the space, the deeper the detection . ${ }^{[10]}$

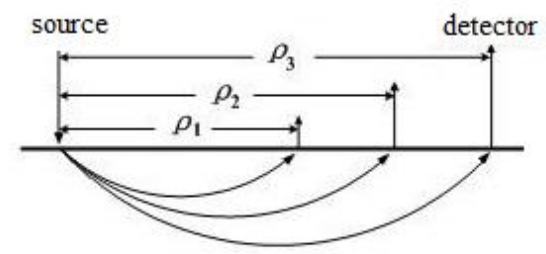

Fig.1.The relationship of detecting depth and the space $(\rho)$

Meat mainly contains protein, moisture, fat and little of extractum. In the absorption characteristics of wavelength range, protein such as myoglobin and hemoglobin in the pork organization overlaps the moisture. Therefore, spectrum of pork is both a multicomponent absorption and scattering effect. As shown in figure 2 , in the wavelength range of $600 \mathrm{~nm}$ and $1700 \mathrm{~nm}$, the absorption peaks of 
moisture are $970 \mathrm{~nm}$ and $1450 \mathrm{~nm}^{[5]}$. By detecting the intensity of diffuse reflectance of each wavelength, we can associate the moisture of meat and the diffuse reflectance, and then build a prediction model related to the content of each component. To accurately express the original information, considering other background material in the pork organization, in this paper, we apply the design of multi-wavelength. Then we made the multiple linear relationships between spectral information parameters measured by the detector and pork moisture content value measured by the physical and chemical methods.

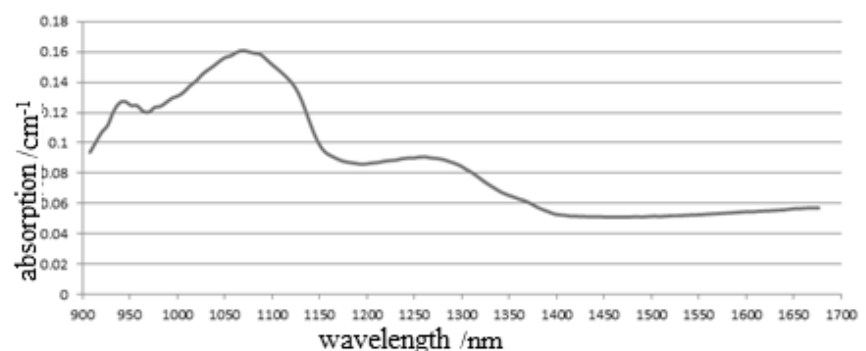

Fig.2.The absorption in the wavelength range of $600 \mathrm{~nm}$ and $1700 \mathrm{~nm}$

\subsection{Measuring device}

We used infrared detection device based on discrete type illuminant, whose LED light source wavelengths are $970 \mathrm{~nm}, 1300 \mathrm{~nm}, 1450 \mathrm{~nm}$ and $1550 \mathrm{~nm}$. The overall structure of the system is as follows:

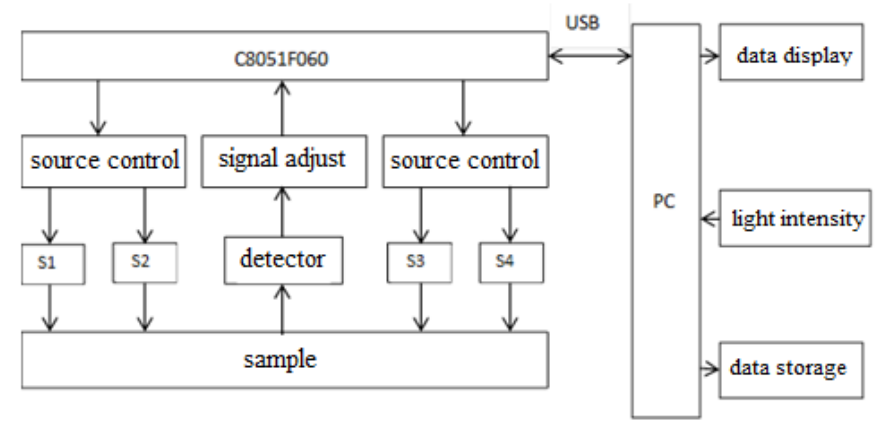

Fig.3.The overall structure of the system

During the procedure of measurement, the main chip C8051F060 receives instructions from the PC. Then the four LED light source, i.e. S1, S2, S3, S4, at four wavelength $970 \mathrm{~nm}, 1350 \mathrm{~nm}, 1450 \mathrm{~nm}, 1550 \mathrm{nmrespectivly,} \mathrm{emitted} \mathrm{light} \mathrm{pulse}$ signal by means of control circuit. The optical signal after scattering and absorption effect of pork samples was measured by the detector, converted to 
analog signals. The analog signals regulate steps such as isolation, amplification straight. When analog electrical signals go through the A/D conversion module of the master control chip, data is converted to spectrum. The data is transformed via USB to the computer .PC software can analysis data, display, storage, etc. In order to eliminate the difference between the sample and the influence of scattering, LED light sources uniformly distribute in the circumference which is $10 \mathrm{~mm}$ away from the detector, shown in figure 4 .

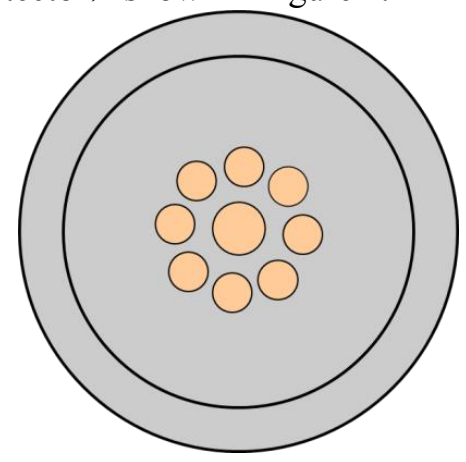

Fig.4. The probe appearance

\section{Experimental materials and method}

\subsection{Experimental materials and equipment}

The samples used in this experiment were longissimus dorsi muscles (LDM), which were obtained from the market with an average weight of $0.5 \mathrm{~kg}$, with a total of 60 samples. The samples were all obtained at $24 \mathrm{~h}$ postmortem from LDM chilled for $24 \mathrm{~h}\left(4^{\circ} \mathrm{C}\right)$. As much as possible, in order to obtain a wide range of characteristic data, each sample is taken from different pig. It is necessary to smooth the surface of pork muscle before the experiment.

Equipment: drying oven(Shanghai jing hong laboratory equipment co., LTD., production DHG29011A type electro thermal constant temperature drying oven, temperature control range: $+10 \sim 200{ }^{\circ} \mathrm{C}$, accuracy: $\pm 1{ }^{\circ} \mathrm{C}$ ), electronic scales(Shanghai Precision and Scientific instrument co., LTD production of YP202N type electronic balance, accuracy: $0.0001 \mathrm{~g}$ ), $100 \mathrm{ml}$ beakers, scalpels, a board, storage bags, our device.

\subsection{Obtaining and analyzing data of samples}

Sample data includes the spectral data and moisture content. Spectral data was collected by the infrared detection device of discrete type illuminant. Experiments 
are conducted in an open environment and room temperature is $22{ }^{\circ} \mathrm{C}$ to $26{ }^{\circ} \mathrm{C}$. The device is coupled directly to the surface of the pork sample. Because the luminous efficiency of LEDs with different wavelengths is not the same, and the diffuse attenuation degree through the pork organization is different, in order to avoid overflow, photoelectric diode detecting signal is adjusted by PC software automatically at the beginning of the experiment. It's necessary to make the light intensity be in a suitable range, and then interval sampling. The data is transformed via USB to the PC software. Do the linear regression with least square method to get $\mathrm{Kn}$ ( each individual wavelengths in the channel). Each $\mathrm{Kn}$ is calculated by five groups of driving voltage DA and the matching output light intensity $\mathrm{AD}$ with linear regression, and then get the diffuse reflectance.

Use Chinese standard drying method (GB/T 9695.15 2008) to measure moisture content of pork samples. For a sample, three pieces each about $10 \mathrm{~g}$ drying moisture measurement, finally take the average.

\subsection{Results and discussion}

There are 51 samples of pork longissimus muscle. All samples are ordered by the moisture contents and renumbered. Samples whose number is 5 or multiples of 5 are classified to validation set, others into learning set. In this way, not only ensure validation set widely enough and avoid the validation set beyond learning set range. According to the classification method, learning set is a total of 41 used to establish the model, accounting for about $80 \%$ of the total samples; the remaining 10 samples as a validation set are used to predict. The quantity of learning set can meet the demands of modeling. The table below is real value of the moisture content measured by Chinese standard method.

Table1. Moisture content of the samples

\begin{tabular}{cccccccccc}
\hline Num & content & Num & content & Num & content & Num & content & Num & content \\
\hline 1 & 73.195 & 13 & 74.571 & 26 & 76.357 & 38 & 76.892 & 5 & 74.085 \\
2 & 73.519 & 14 & 74.621 & 27 & 76.406 & 39 & 76.898 & 10 & 74.408 \\
3 & 73.538 & 16 & 74.853 & 28 & 76.414 & 41 & 76.963 & 15 \\
4 & 74.047 & 17 & 75.206 & 29 & 76.456 & 42 & 77.070 & 20 & 75.743 \\
6 & 74.221 & 18 & 75.277 & 31 & 76.501 & 43 & 77.106 & 25 & 76.312 \\
7 & 74.292 & 19 & 75.544 & 32 & 76.511 & 44 & 77.354 & 30 & 76.501 \\
8 & 74.325 & 21 & 75.938 & 33 & 76.753 & 46 & 77.405 & 35 & 76.804 \\
9 & 74.377 & 22 & 75.962 & 34 & 76.766 & 47 & 77.440 & 40 & 76.948 \\
11 & 74.481 & 23 & 76.056 & 36 & 76.814 & 48 & 77.517 & 45 & 77.372 \\
12 & 74.568 & 24 & 76.132 & 37 & 76.871 & 49 & 77.522 & 50 & 77.533 \\
& & & & & & 51 & & 77.654 & \\
\hline
\end{tabular}


This device with four wavelength detector, can be measured with a total of four diffuse reflectance, the four diffuse reflectance used to determine moisture content of real value by using multiple linear regression model by the least squares method. We trained the learning set and validated test set respectively, learning set predicted results as shown in figure 5 , the $\mathrm{R}^{2}$ and the root mean square error are 0.492 and 0.914 respectively; Validation set predicted results as shown in figure 6 , decision coefficient and the root mean square error are 0.778 and 0.645 respectively.

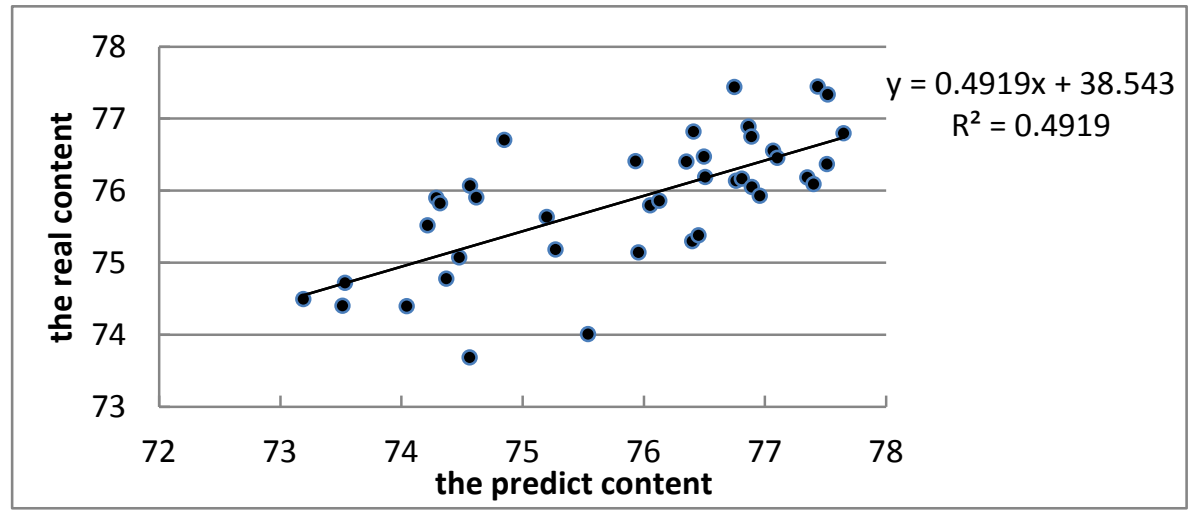

Fig.5. Comparison predicted moisture content by near infrared spectroscopy using the proposed model consisting of fours wavelengths to measured moisture content (the learning set)

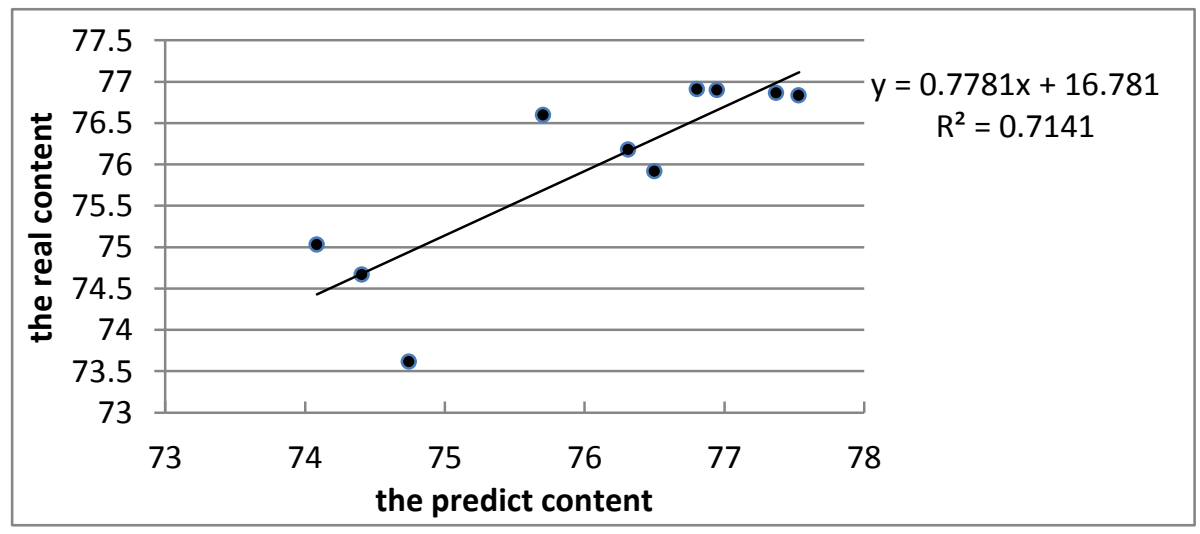

Fig.6. Comparison predicted moisture content by near infrared spectroscopy using the proposed model consisting of fours wavelengths to measured moisture content (the validated test set)

As you can see from the above test, the $\mathrm{R}^{2}$ needs to be improved. The main source of error is the following several aspects:

(1) That the different scattering between different samples of pork, is an important source of error. Pork tissue is the strong scattering medium. Scattering will occur within the organization on the boundary of the discontinuous refractive index, and is related to the structure, structure change, the number and distribution of tissue cells. 
Because of the experimental sample sources united hardly, and directions of fiber of different samples are different, those effect the scattering, namely detection under the influence of thickness of fibers and their distribution.

(2) Heterogeneity of the pork tissue leads to the difference of the scattering and absorption of the different parts[11].The distribution of moisture is not uniform in pork tissues, which caused the moisture content in different areas of the same sample measured by the Chinese standard method are different.

(3) The breeds, growing environment and different slaughter time also have an important influence on the scattering and absorption of light. Tracking information of pork needs to be perfected.

The moisture content of samples which we bought from the market are mainly concentrated in between $74 \%$ and $77 \%$, average from $75.5 \%$ to $76.5 \%$. But the samples whose moisture content is below $74 \%$ or more than $77 \%$ are relatively little. This reduces the accuracy of predicting for the high moisture or low.

\section{Conclusions}

By the experimental results and data analysis, we can see the accuracy of moisture detection remains to be further strengthened. Reasons influencing the accuracy of the experiment are mainly that the tissue of fresh pork meat is not homogeneous, and the directions of fiber are different, and scattering. Fresh meat storage and unscattered sample moisture content data also have a certain influence on the accuracy of predicted results.

\section{Acknowledgment}

This research was supported by the National Key Technology R\&D Program of China (Project No. 2012BAH04B02).

\section{References}

1. Liang Xuefeng, Lv Pingping, Zhang Xuequan. Research and development of the standards for moisture content of pork and beef meat. ISSN 1008-5467, 2001(08):38-43. (in Chinese)

2. Zhu Danshi, Wu Xiaofei, Liu He, Xu Yongxia, LI Jianrong. Effect of fresh meat[J].Science and Technology of Food Industry, 2013(16):363-366. (in Chinese)

3. Prieto Nuria, Roehe R., Lavín Paz, et al. Application of near infrared reflectance spectroscopy to predict meat and meat products quality: A review[J]. Meat Science, 2009, 83(2):175-186.

4. Barbin Douglas F., ElMasry Gamal,Sun Dawen, et al. Non-destructive determination of chemical composition in intact and minced pork using near-infrared hyperspectral imaging [J]. Food chemistry, 2013,138(2):1162-1171 
5. Sun Tong, Xu Huirong, Ying Yibin. Progress in Application of Near Infrared Spectroscopy to Nondestructive On-line Detection of Products/Food Quality[J]. Spectroscopy and Spectral Analysis,2009(01):122-126. (in Chinese)

6. Xu Xia, Cheng Fang, Ying Yibin. Application and Recent Development of Research on Near Infrared Spectroscopy for Meat Quality Evaluation[J]. Spectroscopy and Spectral Analysis,2009(07):1876-1880. (in Chinese)

7. Wen Xing, Wang Zhongyi, Huang Lan. Measurement of myoglobin in pork meat by using steady spatially-resolved spectroscopy [J]. Transactions of the CSAE, 2010, 26(Supp.2): 375 -379. (in Chinese)

8. Zhang Genwei, Wen Xing, Wang Zhongyi, Zhao Dongjie, Huang Lan. Measurement of Pork Tenderness by Using Steady Spatially-Resolved Spectroscopy[J]. Spectroscopy and Spectral Analysis ,2010(10):2793-2796. (in Chinese)

9. JI Ruiqi n, Hung Lan, Liu L i, Wang Zhongyi. Method for Measuring Water Content in Fresh Meat Using Diffusion Reflectance Near Infrared Spectroscopy and Experiment [J]. Spectroscopy and Spectral Analysis, 2008(08):1767-1771.

10.Lai Jiancheng, Li Zhenhua, Wang Qinghua, He Anzhi. System Model of Light Transporting in Biological Tissues and its Application[J]. ACTA PHOTONICA SINICA , 2007, (07): 1312-1317. (in Chinese)

11. Barbin Douglas, Elmasry Gamal, Sun Da-Wen, et al. Near-infrared hyperspectral imaging for grading and classification of pork[J]. Meat Science, 2012,90(1):259-268. 